\title{
A dual frequency-selective bounded real lemma and its applications to IIR filter design*
}

\author{
H.D. Tuan ${ }^{1}$, N.T. Hoang ${ }^{2}$, H.Q. $\mathrm{Ngo}^{3}$, H. Tuy ${ }^{4}$ and B. $\mathrm{Vo}^{2}$
}

\begin{abstract}
Given a transfer function $H(s)$ of order $n$, the celebrated bounded real lemma characterises the untractable semi-infinite programming (SIP) condition $|H(\jmath \omega)|^{2} \leq \gamma^{2} \forall \omega \in R$ of function bounded realness (BR) by a tractable semi-definite programming (SDP). Some recent results generalise this result for the SIP condition $|H(\jmath \omega)|^{2} \leq \gamma^{2} \forall|\omega| \leq \bar{\omega}$ of frequency-selective bounded realness (FSBR). The SDP characterisations are given at the expense of an introduced Lyapunov matrix variable of dimension $n \times n$. As a result, the dimension of the resultant SDPs grows so quickly in respect to the function order, making them much less computationally tractable and practicable. Moreover, they do not allow to formulate synthesis problems as SDPs.

In this paper, a completely new SDP characterizations for general FSBR for all-pole transfer functions is proposed. Our motivation is the design of infinite-impulseresponse(IIR) filters involving a few of simutaneous FSBRs. Our SDP characterizations are of moderate size and free from Lyapunov variables and thus allow to address problems involving transfer functions of arbitrary order. Examples are also provided to validate the effectiveness of the resulting SDP design formulation.

Finally we also raise some issues arising with practicability of SDP for multi-dimensional filter design problems. In particular, any bilinear matrix inequality (BMI) optimization is shown to be solved by a SDP with any prescribed tolerance but the issue is dimensionality of this SDP.
\end{abstract}

\section{INTRODUCTION}

The positive real lemma and its variations such as Kalman-Popov-Yakubovich lemma, bounded real lemmas (see e.g. [1], [14]) are certainly among most

\footnotetext{
*The work is supported by the Australian Research Council under grant ARC Discovery Project 0556174

${ }^{1}$ School of Electrical and Telecommunication Engineering, University of New South Wales, UNSW Sydney, NSW 2052, AUSTRALIA. E-mail: h.d.tuan@unsw.edu.au

${ }^{2}$ Department of Electrical and Electronic Engineering, University of Melbourne, Parkville, Vic 3052, AUSTRALIA-Email: \{t.nguyen,bv\}@ee.unimelb.edu.au

${ }^{3}$ Department of Computer Science and Engineering, State University of New York at Buffalo, 201 Bell Hall, Buffalo, NY 142602000, USA. E-mail: hungngo@cse.buffalo.edu

${ }^{4}$ Institute of Mathematics, 18 Hoang Quoc Viet Rd., Hanoi, VIETNAM; Email htuy@math.ac.vn
}

important results of modern control. They allow to express a computationally untractable SIP constraint of a transfer function $H(s)$ by a computationally tractable SDPs for its state-space realization. For instance, the bounded real (BR) condition $|H(\jmath \omega)|^{2} \leq \gamma^{2} \forall \omega \in R$ of $n$-order transfer function $H(s)$ is characterised by a SDP involving its state-space realization $(A, B, C, D)$ and the Lyapunov matrix function variable of dimension $n \times n$ [1], [14]. As it can be seen, the variable dimension $n \times n$ of the Lyapunov variable, which is equivalent to $n(n+1) / 2$ scalar variables, increases very quickly as the function order $n$ increases moderately. As a consequence, the resultant SDPs are large dimensional and hardly solved by the presently available SDP solvers such as [16]. For instance, a function order $n=100$ already requires the Lyapunov variable of dimension $100 \times 100$, which is equivalent to 5000 scalar variables. The generalised results (see e.g. [3], [7]) for the FSBR $|H(\jmath \omega)|^{2} \leq \gamma^{2} \forall|\omega| \leq \bar{\omega}$ also experience a similar drawback. The SDP formulation of [7] also does not allow to formulate a synthesis problem as a SDP. More exactly, it leads to a bilinear matrix inequality (BMI) formulation for the problem. In [17], we have obtained a new SDP characterisation for the FRPR of discrete-time function $H\left(e^{\jmath \omega}\right)$ of finite impulse response (FIR). Our SDP formulation is of substantially reduced order and its dual formulation does not involve any additional variables and thus open a new way for effective solution of large dimensional digital (discrete) systems. We have implemented in [17] computational examples involving the design of 1200order system arising from digital filter design.

In this paper, we obtain a new FSBR lemma for all-pole functions. They are among the most popular classes in IIR analog filter design, which is the most fundamental problem of signal processing [12]. Like our previous results for discrete-time case, our new SDP characterization is moderate side and its dual formulation is free from slack variables and leads to a very robustly numerical algorithm.

The paper is organised as follows. A new optimisationbased design formulation for the class of all-pole filter is presented next in Section 2. FSBR lemmas and effi- 
cient SDP based solution to the proposed formulation is given in Section 3. An extension to multi-dimensional filters is provided in Section 4 and conclusions are drawn in Section 5.

Notations. For symmetric matrix $X, X \geq 0$ or $X \leq 0$ means $X$ is semi-positive definite or semi-negative definite, respectively. Also, as usual ${ }^{T}$ symbolizes the matrix transposition operator and if matrices $Y$ and $Z$ are of appropriate dimensions then $\langle Y, Z\rangle=\operatorname{Trace}(Y Z)$. The convex hull conv $(C)$ (conic hull cone $(C)$, respectively) of a set $C \subset R^{n}$ is the smallest convex set (smallest convex cone, respectively) containing $C$. The polar cone $C^{*}$ of $C$ is defined as the set $\left\{x \in R^{n}\right.$ : $\langle x, c\rangle \geq 0, \forall c \in C\}$. We refer the reader to [15] for the background of convex analysis. Also $[n / 2]$ is the largest integer not exceeding $n / 2$ for an integer $n$.

\section{Motivation: ALL-POLE ANALOG FILTER DESIGN}

The reader is referred to [5] for a brief introduction for the state of art of analog filter design.

In its plain form, the problem is to design a filter that matches as smoothly as possible an ideal nonimplementable filter of the frequency response 1 at a given passband and zero at a given stopband. Thus the ripple constraints on the passband and stopband, which are in fact particular FSBR, are introduced to attain the smooth filter performance and also high signal-to-noise (SNR) performance in its implementation at the noisy environment.

Thus, the following criteria are set for designing a normalized low pass filter of order $n$ with transfer function $F(s)$ :

(i) Least aggregate squared error over the pass band

$$
\Sigma_{E}=\int_{0}^{1}\left(|F(j \omega)|^{2}-1\right)^{2} d \omega \rightarrow \min
$$

(ii) Given a peak error $0<\delta_{P}<1$, FSBR constraint of magnitude error or ripple constraint over the pass band

$$
-\delta_{P} \leq|F(j \omega)|^{2}-1 \leq \delta_{P}, \forall \omega \in[0,1] .
$$

(iii) Given the stopband bound $\omega_{S}$, and a peak error $\delta_{S}$, FSBR constraints of magnitude error constraint or ripple constraint over the stop band

$$
0 \leq|F(j \omega)|^{2} \leq \delta_{S}, \forall \omega \geq \omega_{S}
$$

For an all-pole analog filter of order $n$, its transfer function $F(s)$ is

$$
|F(j \omega)|^{2}=F(j \omega) F(-j \omega)=\frac{1}{1+P\left(\omega^{2}\right)}
$$

with

$$
P\left(\omega^{2}\right)=\sum_{i=0}^{n} p_{i} \omega^{2 i}, p_{n}>0,
$$

where $\mathbf{p}=\left(p_{0}, p_{1}, p_{2}, \cdots, p_{n}\right)^{T} \in R^{n}$ and of course, for any $\omega$, the inequality $1+P\left(\omega^{2}\right)>0$ must hold.

The filter design problem for all-pole analog filter is now to find the vector $\mathbf{p}$ to minimise the objective (1) subject to constraints (2) and (3).

To begin with, the FSBR constraints (2) and (3) for the all-pole transfer function $F(\jmath \omega)$ are equivalent to the following FSBR for the polynomial $P\left(\omega^{2}\right)$

$$
\begin{gathered}
\Delta_{P L} \leq P\left(\omega^{2}\right) \leq \Delta_{P U}, \forall \omega \in[0,1] \\
\Delta_{S} \leq P\left(\omega^{2}\right), \quad \forall \omega \geq \omega_{S},
\end{gathered}
$$

where

$$
\Delta_{P L}=-\frac{\delta_{P}}{1+\delta_{P}}, \Delta_{P U}=\frac{\delta_{P}}{1-\delta_{P}}, \Delta_{S}=\frac{1-\delta_{S}}{\delta_{S}} .
$$

In view of (6),

$$
\begin{aligned}
& \frac{1}{\left(1+\Delta_{P U}\right)^{2}} \int_{0}^{1} P^{2}\left(\omega^{2}\right) d \omega \leq \int_{0}^{1} \frac{P^{2}\left(\omega^{2}\right)}{\left(1+P\left(\omega^{2}\right)\right)^{2}} d \omega \\
& \leq \frac{1}{\left(1+\Delta_{P L}\right)^{2}} \int_{0}^{1} P^{2}\left(\omega^{2}\right) d \omega,
\end{aligned}
$$

where $\Delta_{P L}$ and $\Delta_{P U}$ are small as defined by (6). Hence, an adequate approximation of $\Sigma_{E}$ in (1) is the convex quadratic function

$$
\int_{0}^{1} P^{2}\left(\omega^{2}\right) d \omega=\mathbf{p}^{\mathbf{T}} Q \mathbf{p}
$$

with $Q$ defined as

$$
\int_{0}^{1}\left[\begin{array}{c}
1 \\
\omega^{2} \\
\vdots \\
\omega^{2 n}
\end{array}\right]\left[\begin{array}{c}
1 \\
\omega^{2} \\
\vdots \\
\omega^{2 n}
\end{array}\right]^{T} d \omega=\left[\begin{array}{cccc}
1 & \frac{1}{3} & \cdots & \frac{1}{2 n+1} \\
\frac{1}{3} & \frac{1}{5} & \cdots & \frac{1}{2 n+3} \\
\vdots & \vdots & \cdots & \vdots \\
\frac{1}{2 n+1} & \frac{1}{2 n+3} & \cdots & \frac{1}{4 n+1}
\end{array}\right]>0 .
$$

To sum up, our filter design problem is reduced to the following quadratic objective minimisation under FSBR constraints

$$
\min _{\mathbf{p} \in R^{n+1}} \mathbf{p}^{T} Q \mathbf{p}:(6),(7) .
$$

In what follows we define the sets of FSBR constraints

$$
\begin{aligned}
& \mathcal{P}(a, b)=\left\{\mathbf{p} \in R^{n+1}: \sum_{i=0}^{n} p_{i} t^{i} \geq 0, \forall t \in[a, b]\right\}, \\
& \mathcal{P}(a)=\left\{\mathbf{p} \in R^{n+1}: \sum_{i=0}^{n} p_{i} t^{i} \geq 0, \forall t \in[a,+\infty]\right\} .
\end{aligned}
$$

Then the optimisation problem (11) is just

$$
\min _{\mathbf{p} \in R^{n+1}} \mathbf{p}^{T} Q \mathbf{p}: \mathbf{p}-\Delta_{P L} e_{1} \in \mathcal{P}(0,1),
$$


$\Delta_{P U} e_{1}-\mathbf{p} \in \mathcal{P}(0,1), \mathbf{p}-\Delta_{S} e_{1} \in \mathcal{P}\left(\omega_{S}^{2}\right)$,

where $e_{1}=\left[\begin{array}{llll}1 & 0 & \cdots & 0\end{array}\right]^{T} \in R^{n+1}$.

Note that the stable transfer function $F(s)$ is found from the optimal solution of the above problem (13)(14) through spectral factorisation for the function [11]

$$
F(s) F(-s)=\frac{1}{1+P\left(-s^{2}\right)}=\frac{1}{1+\sum_{i=0}^{n} p_{i}\left(-s^{2}\right)^{i}} .
$$

\section{FSBR LEMMA AND POLYNOMIAL CURVES}

We have seen that FSBR constraints (2) and (3) for the all-pole transfer function $F(s)$ are in fact equivalent to FSBR ones (6) and (7) for the polynomial $P\left(\omega^{2}\right)=$ $1 /|F(\jmath \omega)|^{2}-1$. As both (6) and (7) are particular cases of FSBRs (12), we now derive SDP characterisation for (12).

There are two interpretations for (12). The first most natural one is its appeared form (12) saying merely that the polynomial $\sum_{i=0}^{n} p_{i} t^{i}$ is positive on the segment $[a, b]$. As firstly shown in [10], it is equivalent to a SDP constraint through the Markov-Lukacs theorem on positive polynomials. However, as we will see in Subsection 3.1, the resulting SDP is of potentially high dimension. The second less visible one is that p belongs to the polar cone of the polynomial curve $\left\{\left(1, t, \ldots, t^{n}\right)^{T}: t \in[a, b]\right\}$. As it will be seen in the Subsection 3.2, it will lead to a SDP description of substantially reduced dimension and result in a very robust numerical algorithm. Let us describe them in order.

\section{A. Markov-Lukacs theorem as a primal FSBR lemma}

The $k$-th order moment matrix is $(k+1) \times(k+1)$ positive semi-definite and defined as [8]

$$
\mathcal{M}_{k}(t)=\left[\begin{array}{c}
1 \\
t \\
\vdots \\
t^{k}
\end{array}\right]\left[\begin{array}{c}
1 \\
t \\
\vdots \\
t^{k}
\end{array}\right]^{T}=\left[\begin{array}{cccc}
1 & t & \cdots & t^{k} \\
t & t^{2} & \cdots & t^{k+1} \\
\vdots & \vdots & \cdots & \vdots \\
t^{k} & t^{k+1} & \cdots & t^{2 k}
\end{array}\right],
$$

and accordingly, the matrix $\mathcal{M}_{k}(y)$ is created from $\mathcal{M}_{k}(t)$ by the variable change

$$
t^{h} \leftarrow y_{h}, h=0,1,2, \cdots, 2 k
$$

i.e. $\mathcal{M}_{k}(y)$ is

$$
\mathcal{M}_{k}\left(y_{0}, y_{1}, \ldots, y_{2 k}\right)=\left[\begin{array}{cccc}
y_{0} & y_{1} & \cdots & y_{k} \\
y_{1} & y_{2} & \cdots & y_{k+1} \\
\vdots & \vdots & \cdots & \vdots \\
y_{k} & y_{k+1} & \cdots & y_{2 k}
\end{array}\right] \text {. }
$$

Define also

$$
\mathcal{M}_{1 k}(t)=t \mathcal{M}_{k}(t)
$$

and accordingly

$$
\mathcal{M}_{1 k}(y) \equiv \mathcal{M}_{\ell k}\left(y_{0}, y_{1}, \ldots, y_{2 k+1}\right)
$$

is created from $\mathcal{M}_{\ell k}(t)$ by the variable change (17) for $h=0,1, \ldots, 2 k+1$.

For instance, it can be easily checked that

$$
\mathcal{M}_{1(k-1)}(y)=\left[\begin{array}{ll}
I_{k \times k} & 0_{k \times 1}
\end{array}\right] \mathcal{M}_{k}(y)\left[\begin{array}{c}
0_{1 \times k} \\
I_{k \times k}
\end{array}\right] \in R^{k \times k}
$$

The role of moment matrices is shortly clarified in the following theorem.

Markov-Lucacs theorem. One has $\mathbf{p} \in \mathcal{P}(a, b)$ if and only if there are matrices $X \geq 0$ and $Z \geq 0$ of size $([n / 2]+1) \times([n / 2]+1)$ such that

$\sum_{i=0}^{n} p_{i} t^{i}=(t-a)\left\langle X, M_{[n / 2]}(t)\right\rangle+(b-t)\left\langle Z, M_{[n / 2]}(t)\right\rangle$

Also, $\mathbf{p} \in \mathcal{P}(a)$ if and only if

$$
\sum_{i=0}^{n} p_{i} t^{i}=(t-a)\left\langle X, M_{[n / 2]}(t)\right\rangle+\left\langle Z, M_{[n / 2]}(t)\right\rangle,
$$

for some $X \geq 0, Z \geq 0$ of size $([n / 2]+1) \times([n / 2]+1)$.

The above theorem follows from the so called snake theorem [8] with a quite complex and long proof involving many mathematical constructs. Actually, the whole chapter 7 of [8] is devoted to its proof! A short and elementary proof for the above version of this famous theorem is provided in the appendix I.

Based on (20) and (21), the primal FSBR lemma for the transfer function $F(s)$ can be easily stated by obtaining the linear constraints in $\mathbf{p}$ and $X, Z$ derived by comparison of terms with the same power $t^{i}$ of $t$ in both sides of (20) and (21). As indicated in the statement of the Markov-Lucacs Theorem, $X$ and $Z$ must also satisfy the SDP constraints $X \geq 0$ and $Z \geq 0$, so each constraint in (14) in $\mathbf{p}$ is in fact equivalently expressed by a LMI constraint in $\mathbf{p}$ and additional variables $X, Z$. As a result, for instance (13)-(14) is reduced to a SDP in $\mathbf{p}$ and 6 additional matrix variables of dimensions $([n / 2]+1) \times([n / 2]+1)$, i.e. the number of variables increases substantially in such a SDP formulation. In the next subsection, we use the polar cone interpretation for the constraint (12) to provide a novel technique, which allows us to solve (13)-(14) by a SDP with a much smaller number of variables. 
B. The convex hull of polynomial curves and dual FSBR lemma

The dual FSBR lemma is based on the following LMI characterizations of the convex hull of (nonconvex) polynomial curve (in $R^{n+1}$ ).

Theorem 1: The conic hull of the polynomial curve $C_{a, b}$ defined as

$$
C_{a, b}=\left\{\left[\begin{array}{llll}
1 & t & \cdots & t^{n}
\end{array}\right]^{T}: t \in[a, b]\right\}
$$

is fully characterised by LMIs: $y \in \operatorname{cone}\left(C_{a, b}\right)$ if and only and it satisfies the LMIs

$$
b \mathcal{M}_{[n / 2]}(y) \geq \mathcal{M}_{1[n / 2]}(y) \geq a \mathcal{M}_{[n / 2]}(y)
$$

The conic hull of the polynomial curve $C_{a}$ defined as

$$
C_{a}=\left\{\left[\begin{array}{llll}
1 & t & \cdots & t^{n}
\end{array}\right]^{T}: t \in[a,+\infty]\right\}
$$

is fully characterised by LMIs: $y \in \operatorname{cone}\left(C_{a}\right)$ if and only if it satisfies the LMIs

$$
\mathcal{M}_{[n / 2]}(y) \geq 0, \mathcal{M}_{1[n / 2]}(y) \geq a \mathcal{M}_{k}(y)
$$

Proof: See the appendix II.

Remark. For $n$ even, by the definition, $\mathcal{M}_{1[n / 2]}$ is a matrix function of $\left(y_{0}, y_{1}, \ldots, y_{n}, y_{n+1}\right)$ and accordingly LMIs (23), (25) are understood for some $y_{n+1}$.

As a further step, from the definition of $\mathcal{P}(a, b)$ and $\mathcal{P}(a)$, it is clear that

$$
\begin{gathered}
\mathcal{P}(a, b)=\left(C_{a, b}\right)^{*}=\left(\operatorname{conv}\left(C_{a, b}\right)\right)^{*} \\
\mathcal{P}(a)=\left(C_{a}\right)^{*}=\left(\operatorname{conv}\left(C_{a}\right)\right)^{*}
\end{gathered}
$$

so (13)-(14) can be written as

$$
\begin{array}{r}
\min _{\mathbf{p}} \mathbf{p}^{T} Q \mathbf{p}: \mathbf{p}-\Delta_{P L} e_{1} \in C_{0,1}^{*}, \Delta_{P U}-\mathbf{p} \in C_{0,1}^{*}, \\
\mathbf{p}-\Delta_{s} e_{1} \in C_{\omega_{s}^{2}}^{*}
\end{array}
$$

where for simplicity of notations we use $C_{0,1}^{*}$ and $C_{\omega_{s}^{2}}^{*}$ to refer $\left(\text { cone } C_{0,1}\right)^{*}$ and $\left(\text { cone } C_{\omega_{s}^{2}}\right)^{*}$, respectively.

The dual problem of (26) is the SDP problem

$$
\begin{gathered}
\max _{y^{(1)}, y^{(2)}, y^{(3)}, \nu} \Delta_{P L} e_{1}^{T} y^{(1)}-\Delta_{P U} e_{1}^{T} y^{(2)}+\Delta_{S} e_{1}^{T} y^{(3)}-\nu: \\
{\left[\begin{array}{cc}
\nu & \left(-y^{(1)}+y^{(2)}-y^{(3)}\right)^{T} \\
-y^{(1)}+y^{(2)}-y^{(3)} & 4 Q
\end{array}\right] \geq 0,}
\end{gathered}
$$$$
\text { (23) with } y^{(1)}, y^{(2)} \text { for } 0 \rightarrow a, 1 \rightarrow b \text {, }
$$$$
\text { (25) with } y^{(3)} \text { for } \omega_{S}^{2} \rightarrow a \text {, }
$$

Furthermore, the optimal solution $p$ of (26) can be directly retrieved from the optimal solution $y^{(i)}$ of (27) by the unique solution of the linear equation system

$$
Q \mathbf{p}=-\frac{1}{2}\left(-y^{(1)}+y^{(2)}-y^{(3)}\right)
$$

TABLE I

DESIGN SPECIFICATIONS AND AGGREGATE SQUARED ERROR PERFORMANCES OF THE FREQUENCY NORMALIZED FILTERS

\begin{tabular}{|c|c|c|c|c|}
\hline \multirow{2}{*}{ Filters } & \multicolumn{4}{|c|}{ Specification details } \\
\cline { 2 - 5 } & $\omega_{S}$ & $\delta_{S}$ & $\delta_{P}$ & $\Sigma_{E}$ \\
\hline \hline $4^{t h}$ order filter & 1.6 & 0.015 & 0.03 & $\mathbf{1 . 0 9} \times \mathbf{1 0}^{-4}$ \\
\hline $5^{t h}$ order filter & 1.5 & 0.001 & 0.02 & $\mathbf{4 . 7 5} \times \mathbf{1 0}^{-\mathbf{5}}$ \\
\hline $6^{t h}$ order filter & 1.4 & 0.004 & 0.014 & $\mathbf{6 . 5 3} \times \mathbf{1 0}^{-\mathbf{6}}$ \\
\hline $7^{t h}$ order filter & 1.35 & 0.003 & 0.010 & $\mathbf{6 . 0 9} \times \mathbf{1 0}^{-\mathbf{7}}$ \\
\hline $8^{t h}$ order filter & 1.3 & 0.0022 & 0.008 & $\mathbf{8 . 0 6} \times \mathbf{1 0}^{-\mathbf{7}}$ \\
\hline $9^{t h}$ order filter & 1.25 & 0.0017 & 0.006 & $\mathbf{1 . 0 6} \times \mathbf{1 0}^{-\mathbf{6}}$ \\
\hline $10^{t h}$ order filter & 1.22 & 0.0012 & 0.005 & $\mathbf{2 . 8 7} \times \mathbf{1 0}^{-\mathbf{7}}$ \\
\hline
\end{tabular}

Let us emphasise the advantage of the analytical dual SDP formulation (27). Unlike the primal SDP (26), the dual SDP (27) involves variables of dimension $n+1$ and of moderate dimension so it can be effectively solved by any existing SDP software no matter how $n$ can be large.

The below theorem is now at hand.

Theorem 2: Given the design specifications: stop frequency $\omega_{S}$, pass band and stop band ripple constraints $\delta_{P}$ and $\delta_{S}$, the optimal filter of order $n$ in the sense of least aggregate squared error over the pass band $\Sigma_{E}$ in (1) has the transfer function $F(s)$ resulting from spectral factorization for the function (15), where the coefficients $p_{0}, p_{1}, \cdots, p_{n}$ are derived from the optimal solution $y^{(i)}, i=1,2,3$ of $\operatorname{SDP}(27)$ from the linear equations (28).

\section{Numerical Illustration}

We examine our design formulation via the designs of a number of analog filters using SDP (27). Design parameters are given in Table 1 where the resultant design objectives are written in boldface. Magnitude responses of designed $10^{\text {th }}$ order filters is depicted in Figures 1 . By the table 2, we can see that reduction in the number of scalar variables achieved by (27) gets better fast as the order of the desired filter increases.

Table 3 (with the objective performances of filters stressed in boldface) gives performances of the proposed filters and the Chebysev ones. In the case of the $4^{t h}$ order filter, gain of $0.004 / 0.030=13.3 \%$ in ripple constraint made by the Chebyshev filter results in $0.002 / 0.015=13.3 \%$ loss in aggregate error. For the $5^{\text {th }}$ and $6^{\text {th }}$ filters, that is $15 \%$ versus $36.5 \%$ and $36.4 \%$ versus $51.4 \%$. Thus, in all the three cases our proposed formulation offers considerable aggregate error reduction over the pass band. The sacrifice of the error peak trades off well with the improvement of pass band aggregate error. This subsection together with the 
TABLE II

NUMBER OF SCALAR VARIABLES OF DIFFERENT DESIGN FORMULATIONS

\begin{tabular}{|c|c|c|}
\hline \multirow{2}{*}{ Filters } & \multicolumn{2}{|c|}{ Number of scalar variables } \\
\cline { 2 - 3 } & $(27)$ & $(13)-(14)$ \\
\hline \hline $5^{t h}$ order filter & 19 & 43 \\
\hline $6^{t h}$ order filter & 22 & 56 \\
\hline $7^{t h}$ order filter & 25 & 69 \\
\hline $8^{t h}$ order filter & 28 & 85 \\
\hline $9^{t h}$ order filter & 31 & 101 \\
\hline $10^{t h}$ order filter & 34 & 120 \\
\hline
\end{tabular}

\begin{tabular}{|c|c|c|c|c|}
\hline \multirow{2}{*}{ Designed Filters } & \multicolumn{4}{|c|}{ Specification details } \\
\cline { 2 - 5 } & $\omega_{S}$ & $\delta_{S}$ & $\delta_{P}$ & $\Sigma_{e}$ \\
\hline \hline Prop. 4-order filter & 1.6 & 0.03 & 0.034 & $\mathbf{0 . 0 1 3}$ \\
\hline Cheb. 4-order filter & 1.6 & 0.03 & $\mathbf{0 . 0 3 0}$ & 0.015 \\
\hline Prop. 5-order filter & 1.5 & 0.015 & 0.020 & $\mathbf{0 . 0 0 6 3}$ \\
\hline Cheb. 5-order filter & 1.5 & 0.015 & $\mathbf{0 . 0 1 7 4}$ & 0.0086 \\
\hline Prop. 6-order filter & 1.41 & 0.01 & 0.015 & $\mathbf{0 . 0 0 3 5}$ \\
\hline Cheb. 6-order filter & 1.41 & 0.01 & $\mathbf{0 . 0 1 1}$ & 0.0053 \\
\hline
\end{tabular}

TABLE III

AGGREGATE ERROR OVER THE PASS BAND VERSUS PASS BAND RIPPLE PERFORMANCES

last one consolidate our motivation and validate of our design formulations.

\section{EXTENSIONS TO MULTI-DIMENSIONAL FILTER DESIGN: POTENTIAL DIFFICULTIES}

As we can easily see, the key step for converting the optimization problem (11) to the SDP problem (27) is the exact LMI description (23) for the convex hull of the nonconvex set $C_{a, b}$. Particularly, it also implies that the following univariate polynomial optimization problem of nonconvex optimization

$$
\min _{t \in[a, b]} \sum_{i=0}^{n} c_{i} t^{i}
$$

which is the same as

$$
\min _{y \in \operatorname{conv} C_{a, b}} c^{T} y, c=\left(c_{0}, c_{1}, \ldots, c_{n}\right)^{T}
$$

and thus according to Theorem 1 is solved by SDP

$$
\min _{y \in R^{n+1}} c^{T} y:(23), y_{0}=1
$$

This fact has been mentioned in [9] for $n$ odd in a quite different setting.

On the other hand, it can be shown that the problem of multi-dimensional filter design will involve semiinfinite constraints like

$\mathbf{p} \in R^{N}: \sum_{\alpha} p_{\alpha} \prod_{i} x_{i}^{\alpha_{i}} \geq 0 \forall x_{i} \in\left[a_{i}, b_{i}\right], i=1,2, \ldots, n$.

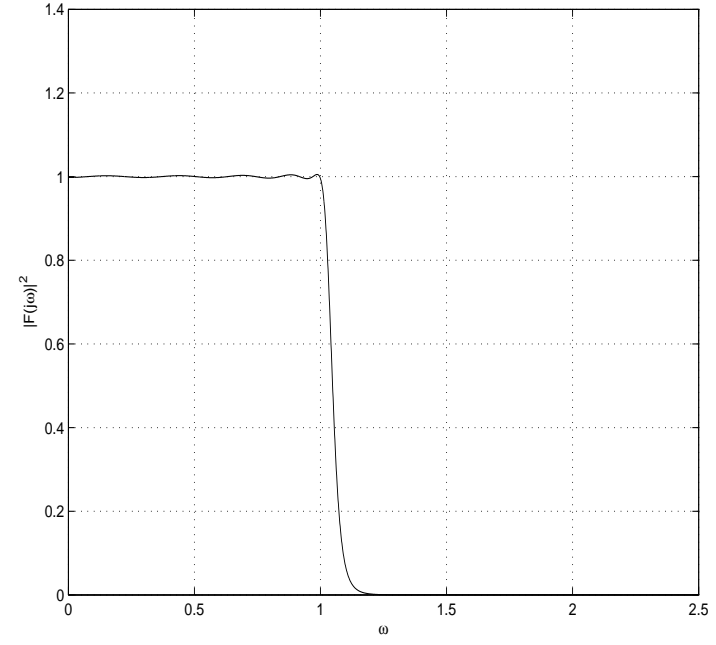

Fig. 1. Magnitude response of the designed $10^{\text {th }}$ order filter

Thus a natural question is whether we can describe the convex hull of the set

$$
\begin{aligned}
& \mathcal{C}=\left\{\left[\begin{array}{llllllllll}
1 & x_{1} & \ldots & x_{n} & x_{1}^{2} & x_{1} x_{2} & \ldots & x_{n}^{2} & \ldots & x_{n}^{k}
\end{array}\right]^{T}:\right. \\
& \left.g_{\ell}(x) \geq 0, \ell=1,2, \ldots, L\right\} \subset R^{N}
\end{aligned}
$$

where $g_{\ell}$ are polynomials in $x=\left(x_{1}, x_{2}, \ldots, x_{n}\right)^{T} \in$ $R^{n}$ :

$$
g_{\ell}(x)=\sum_{\alpha} g_{\ell \alpha} x^{\alpha}, x^{\alpha}=\prod_{i} x_{i}^{\alpha_{i}}, \sum_{i} \alpha_{i}<+\infty .
$$

In what follows we use the following variable changes

$$
x^{\alpha}=\prod_{i} x_{i}^{\alpha_{i}} \leftrightarrow y_{\alpha}=y_{\alpha_{1} \alpha_{2} \ldots \alpha_{n}} \quad \forall \alpha
$$

The $n$-dimensional moment matrices are defined as follows

$$
\begin{aligned}
\mathcal{M}_{1}(x) & =\left[\begin{array}{c}
1 \\
x_{1} \\
\ldots \\
x_{n}
\end{array}\right]\left[\begin{array}{c}
1 \\
x_{1} \\
\ldots \\
x_{n}
\end{array}\right]^{T} \\
& =\left[\begin{array}{ccccc}
1 & x_{1} & x_{2} & \ldots & x_{n} \\
x_{1} & x_{1}^{2} & x_{1} x_{2} & \ldots & x_{1} x_{n} \\
x_{2} & x_{1} x_{2} & x_{2}^{2} & \ldots & x_{2} x_{n} \\
\ldots & \ldots & \ldots & \ldots & \ldots \\
x_{n} & x_{1} x_{n} & x_{2} x_{n} & \ldots & x_{n}^{2}
\end{array}\right](36)
\end{aligned}
$$




$$
\begin{gathered}
\mathcal{M}_{2}(x)=\left[\begin{array}{c}
1 \\
x_{1} \\
\ldots \\
x_{n} \\
x_{1}^{2} \\
x_{1} x_{2} \\
\ldots \\
x_{1} x_{n} \\
x_{2}^{2} \\
x_{2} x_{3} \\
\ldots \\
x_{n}^{2}
\end{array}\right]\left[\begin{array}{c}
1 \\
x_{1} \\
\ldots \\
x_{n} \\
x_{1}^{2} \\
x_{1} x_{2} \\
\ldots \\
x_{1} x_{n} \\
x_{2}^{2} \\
x_{2} x_{3} \\
\ldots \\
x_{n}^{2}
\end{array}\right]= \\
{\left[\begin{array}{cccccc}
1 & x_{1} & & & \\
x_{1} & x_{1}^{2} & \ldots & & & \\
\ldots & \ldots & x_{n} & x_{1}^{2} & \ldots & \\
x_{n}^{2} & x_{1} x_{n}^{2} & \ldots & x_{1}^{3} & \ldots & x_{n}^{2} x_{n}^{2} \\
\ldots & x_{n}^{3} & x_{1}^{2} x_{n}^{2} & \ldots & \ldots & x_{n}^{4}
\end{array}\right]}
\end{gathered}
$$

and so all. Clearly, all moment matrices $\mathcal{M}_{i}(x)$ are positive semi-definite.

Accordingly, one can define

$$
\mathcal{M}_{k i}(x)=g_{k}(x) \mathcal{M}_{i}(x)
$$

and $\mathcal{M}_{k i}(y)$ are created from $\mathcal{M}_{k i}(x)$ through the variable change (35).

Theorem 3: Suppose that the set $\mathcal{C}$ defined by (33) is compact.

Then the convex hull $\operatorname{conv}(\mathcal{C})$ of $\mathcal{C}$ can be analytically described by LMIs with any prescribed tolerance $\epsilon$, i.e. one can show a convex set $\operatorname{conv}_{\epsilon} \mathcal{C}$ defined analytically by LMIs and satisfying

$$
\operatorname{conv}(\mathcal{C}) \subset \operatorname{conv}_{\epsilon} \mathcal{C} \subset \operatorname{conv}(\mathcal{C})+\epsilon O_{N},
$$

where $O_{N}$ is the unit ball in $R^{N}$. One of such convex set $\operatorname{conv}_{\epsilon} \mathcal{C}$ is described by

$$
\begin{array}{r}
\operatorname{conv}_{\epsilon} \mathcal{C}=\operatorname{Proj}\left\{y: \mathcal{M}_{\bar{N}}(y) \geq 0, \mathcal{M}_{\ell(\bar{N}-1)}(y) \geq 0,\right. \\
\ell=1,2, \ldots, L\}
\end{array}
$$

for some $\bar{N}$.

Proof: See the appendix III.

An immediate consequence of the above theorem is the following result on solvability of BMIs, which frequently arise in control synthesis (see e.g. [2]), by SDPs.

Proposition 1: Suppose that the set

$$
\begin{aligned}
\mathcal{C}=\{\tilde{x}= & {\left[\begin{array}{lll}
x_{1} & \ldots & x_{n}
\end{array}\right]: } \\
& \left.G_{\ell}(x) \geq 0, \ell=1,2, \ldots, L\right\} \subset R^{n}
\end{aligned}
$$

is compact, where $G_{\ell}(x) \geq 0$ are BMIs in $x$, i.e. $G_{\ell}$ admits the form

$$
G_{\ell}(x)=G_{\ell 0}+\sum_{i, j} x_{i} x_{j} G_{\ell i j}
$$

Then the convex hull $\operatorname{conv}(\mathcal{C})$ of $\mathcal{C}$ can be analytically described by SDPs with any prescribed tolerance $\epsilon$, i.e. one can show a convex set $\operatorname{conv}_{\epsilon} \mathcal{C}$ defined analytically by SDPs and satisfying (38). Consequently, any BMI optimization problem

$$
\min _{x}\langle c, x\rangle: G_{\ell}(x) \geq 0, \ell=1,2, \ldots, L
$$

can be solved by an SDP with any prescribed tolerance.

Proof: By the Sylvester's criterion, the matrix $G_{\ell}(x)$ is positive-semidefinite if and only if its determinant and principle minors are nonnegative. Obviously, these are polynomials in $x$ and thus the constraint $G_{\ell}(x) \geq 0$ are equivalent to an analytically described polynomial constraints and the above proposition is a direct consequence of Theorem 3.

However, in sharp contrast to the one-dimensional case, there is no closed form for predicting of the highest order $\bar{N}$ of the moment matrices. Actually, $\bar{N}$ is very potentially high. Even for moderate $\bar{N}$ (so we would have a relaxed problem) the resultant SDPs are already very high dimensional and unlikely solved by the existing SDP solvers. Some techniques handling this issue has been initialled in [6].

\section{Conclusions}

The paper has cast a new design of all-pole analog filter into a convex optimization problem, which is based on a new version of FSBR lemmas. Our proposed design is really practical and can be a more appropriate alternative to the classical filters in some actual analog filtering contexts. We have also developed a general framework for SDP applications to multi-dimensional problems and analysed some potential difficulties with their practicability.

\section{APPENDIX I: AN ELEMENTARY PROOF OF MARKOV-LUCACS THEOREM}

For convenience, we deal with polynomials that are non-negative on $[0, \infty)$. One can turn $f(t)$ non-negative on $[a, b]$ into $\bar{f}(t)$ nonnegative on $[0, \infty)$ with the so-called Goursat transform.

Definition 1: Given a polynomial $f(t)$ of degree $n$ and an interval $[a, b]$, the Goursat transform of $f$ on $[a, b]$ is defined as follows.

$$
\bar{f}(t)=(1+t)^{n} f\left(\frac{b+a t}{1+t}\right) .
$$

Lemma 1: (Variation of Goursat's Lemma) $f(t)$ is non-negative on $[a, b]$ if and only if $\bar{f}(t)$ is non-negative on $[0, \infty)$.

Proof. Note that $t \in[0, \infty)$ implies $\frac{b+a t}{1+t} \in[a, b]$, hence the nonnegativity of $f$ on $[a, b]$ implies the non-negativity of $\bar{f}$ on $[0, \infty)$. 
Conversely, suppose $\bar{f}$ is non-negative on $[0, \infty)$. For any $t \in(a, b]$ we can write

$$
f(t)=f\left(\frac{b+a \frac{b-t}{t-a}}{1+\frac{b-t}{t-a}}\right)=\frac{\bar{f}\left(\frac{b-t}{t-a}\right)}{\left(1+\frac{b-t}{t-a}\right)^{n}} \geq 0
$$

since $t \in(a, b]$ implies $\frac{b-t}{t-a} \in[0, \infty)$.

Lastly, $f(a) \geq 0$ by continuity.

Lemma 2: Any $\bar{f}(t)$, which is nonnegative in $[0, \infty)$, can be written in the form

$$
\bar{f}(t)(t)=p^{2}(t)+t q^{2}(t),
$$

where $p(t)$ and $q(t)$ are polynomials such that the degree of each of $p^{2}(t)$ and $t q^{2}(t)$ is of the degree of $\bar{f}(t)$ at most.

Proof: Suppose $f_{1}(t)=p_{1}^{2}(t)+t q_{1}^{2}(t)$, and $f_{2}(t)=p_{2}^{2}(t)+t q_{2}^{2}(t)$, then

$$
\begin{aligned}
f_{1}(t) f_{2}(t)= & \left(p_{1}^{2}(t)+t q_{1}^{2}(t)\right)\left(p_{2}^{2}(t)+t q_{2}^{2}(t)\right) \\
= & \left(p_{1}(t) p_{2}(t)+t q_{1}(t) q_{2}(t)\right)^{2} \\
& +t\left(q_{1}(t) p_{2}(t)-p_{1}(t) q_{2}(t)\right)^{2} .
\end{aligned}
$$

Thus, if we can write $\bar{f}(t)$ as a product of non-trivial factors each of which has the desired form, then $\bar{f}$ itself has the desired form by induction.

Since $\bar{f}(t)$ is non-negative on $[0, \infty)$, every positive root of $\bar{f}$ must have even multiplicity. Hence, the factors corresponding to positive roots have the form $k^{2}(t)$ for some polynomial $k(t)$. Since $k^{2}(t)=$ $k^{2}(t)+t \times 0^{2}$, these factors have the right format.

The factors corresponding to non-positive roots have also the right form: $(t+c)=\sqrt{c}^{2}+t \times 1^{2}$. Also, the factors corresponding to complex roots in conjugate pairs:

$(t-c-i d)(t-c+i d)=\left(-\sqrt{c^{2}+d^{2}}+t\right)^{2}+t\left(\sqrt{2 \sqrt{c^{2}+d^{2}}-2 c}\right)^{2}$ have the desired form.

We are now in position to prove the following version of the original Markov-Lukacs theorem [8].

Lemma 3: Let $f(t)$ be a polynomial of degree $n$ which is nonnegative on $[a, b]$. Then, $f(t)$ admits the following representation:

$$
f(t)=(t-a)\left(p_{1}^{2}(t)+p_{2}^{2}(t)\right)+(b-t)\left(q_{1}^{2}(t)+q_{2}^{2}(t)\right)
$$

where $p_{i}(t)$ and $q_{i}(t)$ are polynomials, and each of the four terms $(t-a) p_{i}^{2}(t),(b-t) q_{i}^{2}(t)$ has degree $n$ at most.

\section{Proof We have}

$$
\begin{aligned}
f(t) & =\frac{(t-a)^{n}}{(b-a)^{n}} \bar{f}\left(\frac{b-t}{t-a}\right) \\
& =\frac{(t-a)^{n}}{(b-a)^{n}}\left[p^{2}\left(\frac{b-t}{t-a}\right)+\frac{b-t}{t-a} q^{2}\left(\frac{b-t}{t-a}\right)\right]
\end{aligned}
$$

which leads to the format

$$
f(t)= \begin{cases}p^{2}(t)+(b-t)(t-a) q^{2}(t) & n \text { is even } \\ (t-a) p^{2}(t)+(b-t) q^{2}(t) & n \text { is odd }\end{cases}
$$

Clearly, for $n$ odd, the format (46) is a particular case of (46).

But for $n$ even, like [4, proof of Lemma 1] it can be immediately checked that (46) gives

$$
\begin{aligned}
(b-a) f(t)= & (t-a) f(t)+(b-t) f(t) \\
= & (t-a)\left(p^{2}(t)+(b-t)^{2} q^{2}(t)\right) \\
& +(b-t)\left(p^{2}(t)+(t-a)^{2} q^{2}(t)\right)
\end{aligned}
$$

i.e. the format (45) again.

Proof of Markov Lukacs theorem: Clearly, (45) is a particular case of (20). On the other hand, the RHS of (20) is a polynomial in $t$, which is nonnegative on $[a, b]$ so it must admit the representations like (45). Therefore, (20) is equivalent to (45).

\section{APPENDIX II: THE PROOF OF THEOREM 1}

First, using the variable change (17) at both sides of (20), (21) leads to the next lemma.

Lemma 4: If $P(t)=\sum_{i=0}^{n} p_{i} t^{i}$ admits the representation (20) for some matrices $X \geq 0, Z \geq 0$ then for every $y=$ $\left[\begin{array}{llll}y_{0} & y_{1} & \cdots & y_{n}\end{array}\right]^{T} \in R^{n}+1$,

$$
\begin{aligned}
\mathbf{p}^{T} y= & \left\langle X, \mathcal{M}_{1[n / 2]}(y)-a \mathcal{M}_{[n / 2]}(y)\right\rangle \\
& +\left\langle Z, b \mathcal{M}_{[n / 2]}(y)-\mathcal{M}_{1[n / 2]}(y)\right\rangle .
\end{aligned}
$$

If $P(t)=\sum_{i=0}^{n} p_{i} t^{i}$ admits the representation (20) for some matrices $X \geq 0, Z \geq 0$ then for every $y=\left[\begin{array}{llll}y_{0} & y_{1} & \cdots & y_{n}\end{array}\right]^{T} \in R^{n+1}$,

$$
\mathbf{p}^{T} y=\left\langle X, \mathcal{M}_{1[n / 2]}(y)-a \mathcal{M}_{[n / 2]}(y)\right\rangle+\left\langle Z, \mathcal{M}_{[n / 2]}(y)\right\rangle .
$$

Proof of Theorem 1. For the first part, the conic hull of $C_{a, b}$ is obviously characterised by LMIs (23) if we can show that the convex hull of $C_{a, b}$ is characterized by LMIs (23) and $y_{0}=1$. In what follows, set $k=[n / 2]$.

Suppose $\operatorname{conv} C_{a, b}$ is the set of $y$ with $y_{0}=1$ satisfying LMIs (23), which is obviously convex. Then $C_{a, b} \subset \operatorname{conv} C_{a, b}$ because each $y=\left[\begin{array}{llll}1 & t & \cdots & t^{n}\end{array}\right]^{T} \in C_{a, b}$ satisfies LMIs (23):

$$
\begin{gathered}
\mathcal{M}_{k}(y)=\mathcal{M}_{k}(t) \geq 0, \\
b \mathcal{M}_{k}(y)-\mathcal{M}_{1 k}(y)=(b-t) \mathcal{M}_{k}(t) \geq 0, \\
\mathcal{M}_{1 k}(y)-a \mathcal{M}_{k}\left(y=(t-a) \mathcal{M}_{k}(t) \geq 0 .\right.
\end{gathered}
$$

From the definition of the convex hull,

$$
\operatorname{conv}\left(C_{a, b}\right) \subset \operatorname{conv} C_{a, b}
$$

follows.

It remains to show

$$
\operatorname{conv} C_{a, b} \subset \operatorname{conv}\left(C_{a, b}\right) .
$$

For that, let the support function [15] for any $A \subset R^{n+1}$ be defined as

$$
s(A, \mathbf{p})=\sup _{y \in A}\langle y, \mathbf{p}\rangle \forall \mathbf{p} \in R^{n+1} .
$$

So

$$
s\left(C_{a, b}, \mathbf{p}\right)-\sum_{i=0}^{n} p_{i} t^{i} \geq 0 \forall t \in[a, b]
$$

and according to Markov Lukacs theorem for each $\mathbf{p}$ there are $X_{\mathbf{p}} \geq 0$ and $Z_{\mathbf{p}} \geq 0$ such that

$s\left(C_{a, b}, \mathbf{p}\right)-\sum_{i=0}^{n} p_{i} t^{i}=(t-a)\left\langle X_{\mathbf{p}}, M_{k}(t)\right\rangle+(b-t)\left\langle Z_{\mathbf{p}}, M_{k}(t)\right\rangle$.

By Lemma 4, it is then true that whenever $y=\left(1, y_{1}, \ldots, y_{n}\right)^{T} \in$ conv $C_{a, b}$ (i.e. $y$ satisfies LMIs (23))

$$
\begin{aligned}
s\left(C_{a, b}, \mathbf{p}\right)-\sum_{i=0}^{n} y_{i} p_{i}= & \left\langle X_{\mathbf{p}}, \mathcal{M}_{1 k}(y)-a \mathcal{M}_{k}(y)\right\rangle \\
& +\left\langle Z_{\mathbf{p}}, b \mathcal{M}_{k}(y)-\mathcal{M}_{1 k}(y)\right\rangle \\
\geq & 0
\end{aligned}
$$

implying $s\left(C_{a, b}, \mathbf{p}\right) \geq s\left(\operatorname{conv} C_{a, b}, \mathbf{p}\right) \forall \mathbf{p} \in R^{n+1}$ or equivalently $\operatorname{conv} C_{a, b} \subset \operatorname{conv}\left(C_{a, b}\right)$ [15].

The proof for the second part is similar.

Remark. As mentioned in the Remark after Theorem 1, when $n$ is even, $\mathcal{M}_{1[n / 2]}(y)$ depends on $\left(y_{0}, y_{1}, \ldots, y_{n+1}\right)$ and the representation (20) implies (47) for all $\left(y_{0}, y_{1}, \ldots, y_{n+1}\right)$. 


\section{APPENDIX III: THE PROOF FOR THEOREM 3}

For any $+\infty>\left|C_{k}\right|:=\max _{\tilde{x} \in C_{k}}\|\tilde{x}\|$, it is obvious that $C_{k} \subset$ $\left|C_{k}\right| . O_{N}$.

Take $\mu:=\epsilon / 4\left|C_{k}\right|$ and let $\left\{p^{(i)}: p^{(i)} \in R^{N},\left\|p^{(i)}\right\|=1, i=\right.$ $1,2, \ldots, \bar{N}\}$ be any $\mu$-net of the unit ball in $R^{N}$, i.e. for any $\|p\|=1$ there is $p^{i}$ such that

$$
\left\|p-p^{(i)}\right\| \leq \mu
$$

It follows from [13] that there are $U^{(i)} \geq 0$ and $V^{(i \ell)} \geq 0$ such that

$$
\begin{array}{r}
s\left(C_{k}, p^{(i)}\right)-\left\langle p^{(i)}, \tilde{x}\right\rangle+\epsilon / 2= \\
\left\langle U^{(i)}, M_{\bar{N}}(x)\right\rangle+\sum_{\ell=1}^{L}\left\langle V^{(i \ell)}, M_{\ell(\bar{N}-1)}(x)\right\rangle,
\end{array}
$$

where $M_{\ell(\bar{N}-1)}(x)=g_{\ell}(x) M_{\bar{N}-1}$ for some $\bar{N}<+\infty$.

Therefore, for $\overline{\left\langle p^{(i)}, y\right\rangle}$ defined from $\left\langle p^{(i)}, \tilde{x}\right\rangle$ by the variable change (35) one has

$$
\begin{aligned}
& s\left(C_{k}, p^{(i)}\right)-\overline{\left\langle p^{(i)}, y\right\rangle}+\epsilon / 2 \\
& \left\langle U^{(i)}, M_{\bar{N}}(y)\right\rangle+\sum_{\ell=1}^{L}\left\langle V^{(i \ell)}, M_{\ell(\bar{N}-1)}(y)\right\rangle .
\end{aligned}
$$

Now, for

$\operatorname{conv}_{\epsilon} C_{k}:=\operatorname{Proj}_{R^{N}}\left\{y: M_{\bar{N}}(y) \geq 0, M_{\ell(\bar{N}-1)}(y) \geq 0\right\} \cap\left|C_{k}\right| . O_{N}$

one has

$$
\begin{array}{r}
s\left(C_{k}, p^{(i)}\right)-\left\langle p^{(i)}, \bar{y}\right\rangle+\epsilon / 2=s\left(C_{k}, p^{(i)}\right)-\overline{\left\langle p^{(i)}, y\right\rangle}+\epsilon / 2 \geq 0 \\
\forall \bar{y} \in \operatorname{conv}_{\epsilon} C_{k} .
\end{array}
$$

Then for each $p \in O_{N}$ there is $p^{(i)} \in O_{N}$ satisfying (49), so whenever $\bar{y} \in \operatorname{conv}_{\epsilon} C_{k}$,

$$
\begin{aligned}
s\left(C_{k}+\epsilon O_{N}, p\right)-\langle p, \bar{y}\rangle & = \\
s\left(C_{k}, p\right)-\langle p, \bar{y}\rangle+\epsilon & = \\
\left(s\left(C_{k}, p\right)-s\left(C_{k}, p^{(i)}\right)\right)+\left(s\left(C_{k}, p^{(i)}\right)-\left\langle p^{(i)}, \bar{y}\right\rangle\right) & \\
+\left(\left\langle p^{(i)}, \bar{y}\right\rangle-\langle p, \bar{y}\rangle\right)+\epsilon & \geq \\
-2 \mu\left|C_{k}\right|+c\left(C_{k}, p^{(i)}\right)-\left\langle p^{(i)}, \bar{y}\right\rangle+\epsilon & = \\
c\left(C_{k}, p^{(i)}\right)-\left\langle p^{(i)}, \bar{y}\right\rangle+\epsilon / 2 & \geq 0
\end{aligned}
$$

implying $\operatorname{conv}_{\epsilon} C_{k} \subset \operatorname{conv}\left(C_{k}\right)+\epsilon . O_{N}$, showing (38).

\section{REFERENCES}

[1] B. Anderson, S. Vongpanitlerd, Network analysis and synthesis: a modern system theory approach, Prentice-Hall, New York, 1973.

[2] P. Apkarian, H.D. Tuan, Concave programming in control theory, J. of Global Optimization 15(1999), 343-370.

[3] T. Davidson, T. Luo, and J. Sturm, "Linear matrix inequality formulation of spectral mask constraints with applications to FIR filter design", IEEE Trans. on Signal Processing 50(2002), 2702-2715.

[4] D. Henrion, J. Lasserre, LMIs for constrained polynomial interpolation with application in trajectory planning, Systems and Control Letters 55 (2006), 473-477.

[5] N.T. Hoang, H.D. Tuan, B. Vo, T.Q. Nguyen, Optimized flat filter dsign by robust semi-definite programming, Submitted
[6] T.Q. Hung, H.D. Tuan, B. Vo, T.Q. Nguyen, SDP for 2-D filter design: general formulation and dimension reduction techniques, Proc. of IEEE Inter. Conf. on Acoustics, Speech and Signal Processing (ICASSP 2006),Toulouse, France, May 2006.

[7] T. Iwasaki, S. Hara, H. Yamauchi, Dynamical system design from a control perspective: finite frequency positive-realness approach, IEEE Trans. on Automatic Control 48(2003), 13371354.

[8] M. Krein, A. Nudel'sman The Markov moment problem and extremal problems, Transl. Math. Monographs 50, American Math. Society, 1977.

[9] J. Lasserre, Global optimization with polynomials and the problem of moments, SIAM J. on Optimization 11(2001), 796817.

[10] Y. Nesterov, "Squared functional systems and optimization problem", High performance optimization $(\mathrm{H}$. Frenk et al. Eds), Kluwer Academic, 2000, pp. 405-440.

[11] A. Papoulis, Probability, Random Variables, and Stochastic Processes, Third Edition, MC Graw-Hill.

[12] H. W. Park, C. S. Burrus Digital filter design John Wiley \& Sons, 1987.

[13] M. Putinar, Positive polynomials on compact semi-algebraic sets, Ind. Univ. Math. J. 42(1993), 969-984.

[14] A. Rantzer, On the Kalman-Yakubovich-Popov lemma, Systems \& Control Letters 28(1996), 7-10.

[15] T. Rockafellar, Convex analysis, Princeton, 1970.

[16] J.F. Sturm, "Using SeDuMi 1.02, a MATLAB toolbox for optimization over symmetric cones", Optimization Methods and Software, 11-12(1999), 625-653.

[17] H.D. Tuan, T.T. Son, B. Vo, T.Q. Nguyen, Efficient large-scale filter/filterbank design via LMI characterization of trigonometric curves, in Proc. of IEEE Inter. Conf. on Acoustics, Speech and Signal Processing (ICASSP 05); also to appear in IEEE Trans. on Signal Processing. 